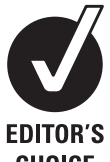

CHOICE

\title{
Trichomonas vaginalis: underdiagnosis in urban Australia could facilitate re-emergence
}

\author{
M Josephine Lusk, ${ }^{1}$ Zin Naing, ${ }^{2}$ Ben Rayner, ${ }^{2}$ Nikolas Rismanto, ${ }^{2}$ \\ Christopher J Mclver, ${ }^{2,3}$ Robert G Cumming, ${ }^{4}$ Kevin McGeechan, ${ }^{4}$ \\ William D Rawlinson, ${ }^{2,3}$ Pam Konecny ${ }^{1,3}$
}

${ }^{1}$ St George Hospital, Kogarah, New South Wales, Australia ${ }^{2}$ Prince of Wales Hospital, Randwick, New South Wales, Australia

${ }^{3}$ University of New South Wales, Randwick, New South Wales, Australia

${ }^{4}$ University of Sydney, Camperdown, New South Wales, Australia

\section{Correspondence to}

Dr M Josephine Lusk, Short Street Clinic, St George Hospital, Kogarah NSW 2217, Australia; luskjo@bigpond.com

The information in this manuscript is not being considered elsewhere for publication. Some of the interim findings of the parent cervicitis study were recently presented by MJL at the Australasian Sexual Health Conference, Brisbane, 3 September 2009.

Accepted 20 October 2009 Published Online First

1 November 2009

\begin{abstract}
Objectives Trichomonas vaginalis (TV) has a low profile in urban sexually transmitted infection (STI) clinics in many developed countries. The objective of this study was to determine the true prevalence of TV in an Australian urban sexual health setting using sensitive molecular diagnostic techniques.

Methods A cross-sectional study investigating the aetiology of cervicitis in women attending two urban sexual health clinics in Sydney, Australia, enrolled 356 consecutive eligible women from 2006 to 2008. The diagnostic yield from the standard clinical practice of discretionary high vaginal wet preparation microscopy in women with suspicious vaginal discharge was compared with universal use of nested PCR for TV of cervical samples.
\end{abstract}

Results TV was detected by PCR in 17/356 women $(4.8 \%, 95 \% \mathrm{Cl} 2.8$ to $7.5 \%)$, whereas only four cases $(1.1 \%, 95 \% \mathrm{Cl} 0.3$ to $2.8 \%)$ were detected by discretionary wet preparation microscopy. Eleven of the 17 women $(p=0.003)$ were of culturally and linguistically diverse background. Additionally, cervicitis was found to be significantly associated with TV, RR 1.66 (1.14 to 2.42), $\mathrm{p}=0.034$.

Conclusions Traditional TV-detection methods underestimate TV prevalence in urban Australia. The TV prevalence of $4.8 \%$ by PCR testing in this study exceeds previously reported urban Australian TV rates of $<1 \%$. An increase in trichomoniasis-associated adverse reproductive outcomes and enhanced HIV transmission poses a salient public health threat. Accordingly, TV warrants a higher profile in urban STI clinic settings in developed countries, and we suggest that priority be given to development of standardised molecular TV detection techniques and that these become part of routine STI testing

\section{INTRODUCTION}

Trichomonas vaginalis (TV) is a sexually transmitted infection (STI) causing significant morbidity worldwide. Trichomonads are highly site-specific protozoan parasites. In women, TV infects the lower urogenital tract, causing superficial vaginal and cervical ulceration. Typical symptoms include frothy yellow discharge, itch, odour, dyspareunia and occasionally vaginal bleeding. Infection of the urethra and paraurethral glands causes dysuria and frequency. ${ }^{1}$ However, at least one-third of infected women may be asymptomatic. ${ }^{2}$ Trichomoniasis has been associated with premature rupture of membranes, ${ }^{3}$ pelvic inflammatory disease (PID), ${ }^{4}$ cervicitis ${ }^{56}$ and enhanced risk of HIV transmission.
Men may present with balanitis, urethral discharge or dysuria, but again, high rates of asymptomatic carriage have been reported. A recent US STI clinic study detected TV in $72 \%$ of male partners of infected females, and of these men, $77 \%$ were asymptomatic. ${ }^{8}$ The duration of TV infection in women may be prolonged for up to $3-5$ years but only about 4 months in men. ${ }^{9}$ The natural history of TV infection is not well defined

Trichomoniasis is the most common curable STI. In 1999, WHO estimated 174 million new cases per year, more than double the number of Chlamydia trachomatis cases and treble the cases of gonorrhoea. The high TV prevalence worldwide is concentrated in developing countries and socio-economically disadvantaged groups, with a dramatic decline in TV rates in some developed countries in the past few decades. ${ }^{10}$ TV prevalence in urban Australia is reportedly low based upon routine wet preparation diagnostic methods. ${ }^{9} 10$ Australian rates of TV peaked in the 1950s at $20-30 \%$ and rapidly declined through the 1960 s and 1970 s to below $1 \%$ in $1990 .{ }^{10}$ This has been attributed to the combination of widespread use of the Nitroimidazoles, and increased surveillance through Papanicolaou smears. ${ }^{9} 10$ This decline and the fact that TV is not a notifiable disease in Australia have led to the present situation where testing for TV has assumed a very low priority in urban Australian settings. An audit of commercial sex workers (CSW) undergoing regular STI screening at a sexual health clinic in Melbourne in 2003 reported a very low incidence of TV at 0.11 per 100 person months. ${ }^{11}$ By contrast, TV prevalence in indigenous Australian women, in remote Northern Territory, was high at $25 \%$ in a self-sampling PCR-method-based study. ${ }^{12}$ The prevalence of TV by PCR among US women of reproductive age was recently found to be $3.1 \%$, with an even lower prevalence of $1.3 \%$ in the subgroup of non-Hispanic white women. ${ }^{13}$

We are conducting a prospective study investigating the prevalence and aetiology of cervicitis using molecular diagnostic techniques in women attending two urban STI clinics in Sydney, Australia. In this paper, we report the prevalence of TV by PCR testing against traditional methods of detection, and compare our findings with previous data from other Australian urban STI clinics.

\section{METHODS}

This study was conducted in two urban Sydney STI clinics from July 2006 to December 2008. Ethics approval was granted by the South Eastern 
Sydney and Illawarra Area Health Service Human Research Ethics Committee and the Sydney South West Area Heath Service Ethics Review Committee (Royal Prince Alfred Hospital Zone).

\section{Subject selection}

Women were eligible for this study if they were 18 years or older, had been sexually active in the previous 3 months and required an internal examination. The parent cervicitis study protocol excluded women if they had been previously enrolled, had clinical PID, had received antibiotics or undergone gynaecological intervention in the previous month, had an intrauterine contraceptive device, were currently menstruating or pregnant, attending for sexual assault or unsuitable for enrolment due to psychosocial ill health or comprehension difficulties. Of a total of 957 consecutive first attendances, 561 women (58.6\%) were ineligible, 40 $(4.2 \%)$ declined, and $356(37.2 \%)$ were enrolled. The most common reasons for ineligibility included examination not required $(30.7 \%)$, antibiotics in previous month $(14.2 \%)$, pregnancy $(8.6 \%)$ and no sex in previous 3 months (8.3\%). Women with clinical PID comprised $3.1 \%$ of ineligible women. This report is an interim analysis of 356 consecutive first attendances of women enrolled in the parent cervicitis study.

\section{Sampling procedure}

A sterile speculum was used to visualise the vagina and cervix. A Gram stain was performed on all cervical and high vaginal swabs (HVS). Due to the nature of the parent study, HVS for wet preparation microscopy was discretionary based upon clinical suspicion of TV infection, in keeping with usual clinical practice, and so not performed for all women. The endocervix was sampled in all women, with a sterile cotton swab (Copan, Brescia, Italy) for Gram stain and bacterial agents. An additional endocervical sterile cotton swab was taken and placed in viral transport medium 199 (GIBCO Invitrogen, Grand Island, New York) and stored at $-70^{\circ} \mathrm{C}$ for subsequent PCR testing for TV by the method described below. Swabs from all women were tested for TV by PCR.

Cervicitis was defined as $>30$ polymorphonuclear cells per high-powered field (PMN/hpf) in at least three non-adjacent fields of cervical mucus on Gram stain of the first endocervical swab. Bacterial vaginosis (BV) was defined by Nugent score from Gram stain of the HVS.

\section{Nucleic acid extraction and PCR amplification}

Swabs were suspended in $500 \mu \mathrm{l}$ of viral transport medium (above) before extraction of the total nucleic acid using a robotic extraction machine (MagNaPure LC, Roche, Germany) applying the Total NA protocol according to the manufacturer's instructions (Roche, Germany). Extracts were stored at $4^{\circ} \mathrm{C}$ before testing within $48 \mathrm{~h}$ of collection.

Detection of TV was performed using a nested PCR. Briefly, the first-round reaction comprised $10 \mu \mathrm{l}$ of template in a $50 \mu \mathrm{l}$ of PCR reaction mixture containing $9 \mu \mathrm{l}$ of nuclease-free water, $25 \mu \mathrm{l}$ of $2 \times$ iScript reaction mix (BioRad, Sydney, Australia), $0.5 \mu \mathrm{M}$ each primer: TricV-OF (5' CTATTGTCGAACATTGGTCTTACCCTC $3^{\prime}$ ) and TricV-OR (5' TCTGTGCCGTCTTCAAGTATGCCC $\left.3^{\prime}\right)^{1415}$ and $1 \mu$ l of iScript RT enzyme (BioRad). (This commercial master mix is used in our laboratory for the amplification of both RNA and DNA templates). Cycling conditions were $50^{\circ} \mathrm{C}$ for $30 \mathrm{~min}$; denaturation at $95^{\circ} \mathrm{C}$ for $15 \mathrm{~min}$, then 35 cycles of $94^{\circ} \mathrm{C}$ for $45 \mathrm{~s}, 57^{\circ} \mathrm{C}$ for $45 \mathrm{~s}$ and $72^{\circ} \mathrm{C}$ for $1 \mathrm{~min}$; a final extension of $7 \mathrm{~min}$ at $72^{\circ} \mathrm{C}$; and a $4^{\circ} \mathrm{C}$ hold. A second round reaction comprised $2 \mu \mathrm{l}$ of first-round product included in a $50 \mu \mathrm{l}$ reaction mixture containing $18 \mu \mathrm{l}$ of nuclease-free water, $25 \mu \mathrm{l}$ of Amplitaq gold PCR Master Mix (Applied Biosystems, Foster City, California, USA) and $0.5 \mu \mathrm{M}$ of each primer: TricV-IF (5' CTCAGTTCGCAAAGGCAGTCCTTGA 3') and TricV-IR (5 GCTTGGAGAGGACATGAACTTCGGA $\left.3^{\prime}\right) .{ }^{14}$ Cycling conditions included denaturation and activation at $95^{\circ} \mathrm{C}$ for $5 \mathrm{~min}, 33$ cycles of: $94^{\circ} \mathrm{C}$ for $20 \mathrm{~s}, 57^{\circ} \mathrm{C}$ for $20 \mathrm{~s}, 72^{\circ} \mathrm{C}$ for $20 \mathrm{~s}$; a final extension at $72^{\circ} \mathrm{C}$ for $10 \mathrm{~min}$ and a $4^{\circ} \mathrm{C}$ hold. PCR products of 206 bp were expected for TV-positives and were visualised by gel electrophoresis. Using positive controls from either cultureproven or molecularly proven sources, sensitivity was assessed by measuring the limit of detection $\left(10^{2}\right.$ copies per reaction) of plasmid constructs of the target sites as previously described ${ }^{14}$ and is estimated to be $95-98 \%$. The specificity was determined as follows: confirmation by probe hybridisation following the PCR amplification, and DNA sequencing performed on PCR products of the first $10 \mathrm{TV}$ positive samples from the study. Nucleotide BLAST on NCBI site (for all 10 samples) confirmed that all DNA sequences produced from sequencing were TV. The results from the above methods confirmed that the assay has $100 \%$ specificity.

\section{Analysis}

We report the prevalence with $95 \%$ CI of TV by traditional methods and PCR methods. Population characteristics of women with and without TV were compared using $\chi^{2}$ testing. p Values $<0.05$ were considered statistically significant. Data were analysed with SAS software SAS Institute (Cary, North Carolina).

\section{RESULTS}

Prevalence of TV by PCR testing was 17/356 (4.8\%, 95\% CI 2.8 to $7.5 \%$ ). Clinical suspicion prompting discretionary wet-mount preparation microscopy identified TV in only 4/356 women $(1.1 \%, 95 \%$ CI 0.3 to $2.8 \%)$. PCR identified a higher percentage of women with TV $(p=0.0003)$.

Of the 17 women positive for TV by PCR testing, only 11 had wet-preparation microscopy performed. The use of discretionary wet preparation was not significantly different in women with and without TV by PCR ( $\mathrm{p}=0.498$ ). Detection by Papanicolaou (Pap) smear occurred in only two of the 11 Pap smears done in women with TV. The mean age of the women with TV, 33.2 years, was not significantly different from the mean age of women without TV, 30.7 years $(p=0.221)$ (table 1$)$. Significantly, $11 / 17$ women with TV $(p=0.003)$ were of culturally and linguistically diverse background and identified consorts from populations of higher TV prevalence overseas (Africa, China, Sri Lanka, South America, Lebanon, Black American). Three women identified rural or 'bush' Australian contacts. Five women were CSW. No cases of TV were indigenous women. Six cases had BV, and three had concurrent STIs (two with Chlamydia and one with HIV and active genital herpes). Dysuria was significantly associated with women with TV $(\mathrm{p}=0.014)$. Prevalence of cervicitis in women without TV was $39 \%$, compared with $65 \%$ $(11 / 17)$ in women with TV, giving a RR of cervicitis in the presence of TV of 1.66 (95\% CI 1.14 to 2.42$) \mathrm{p}=0.034$.

\section{DISCUSSION}

These data show that traditional methods of detection greatly underestimate the prevalence of TV. We report a TV prevalence of $4.8 \%$ by PCR testing, whereas clinical suspicion prompting discretionary wet-preparation microscopy identified TV in only $4 / 356$ women $(1.1 \%)$ in the same study population. The lower 
Table 1 Characteristics of women with and without Trichomonas vaginalis (TV) by PCR

\begin{tabular}{|c|c|c|c|}
\hline Characteristic & $\begin{array}{l}\text { Women with } \\
\text { TV } n=17\end{array}$ & $\begin{array}{l}\text { Women without } \\
\text { TV } N=339\end{array}$ & $\stackrel{p}{\text { Value* }}$ \\
\hline Mean age (years) & 33.4 & 30.7 & 0.221 \\
\hline Culturally and linguistically diverse $†$ & $11(65 \%)$ & $104(31 \%)$ & 0.003 \\
\hline Condoms always & $4(24 \%)$ & $75(22 \%)$ & 0.892 \\
\hline$>1$ partner last 3 months & $6(35 \%)$ & $95(28 \%)$ & 0.516 \\
\hline Commercial sex workers & $5(29 \%)$ & $58(17 \%)$ & 0.195 \\
\hline Concomitant STI‡ & $2(12 \%)$ & $24(7 \%)$ & 0.469 \\
\hline \multicolumn{4}{|l|}{ Signs and symptoms } \\
\hline Dysuria & $7(41 \%)$ & $59(17 \%)$ & 0.014 \\
\hline Vaginal discharge & $11(65 \%)$ & $143(42 \%)$ & 0.067 \\
\hline Bacterial vaginosis§ & $6(35 \%)$ & $79(23 \%)$ & 0.258 \\
\hline
\end{tabular}

${ }^{*} p$ Values $<0.05$ are statistically significant.

tDefined as women identifying at clinic registration as preferring a language other than

English, speaking a language other than English at home, or identifying a non-English ethnic background.

$\neq$ Concomitant chlamydia or gonorrhoea.

$\S$ Defined by Nugent score on Gram stain of high vaginal swab.

case-detection rate by discretionary use of wet-preparation microscopy (and Pap smear) observed here reflects the inadequacy of relying solely upon these methods for detecting TV. Wet-mount microscopy has a sensitivity as low as $52 \%$, ${ }^{16}$ depending on specimen handling, skill of the microscopist and TV organism load in specimen. Additionally, wet-preparation microscopy is not routinely performed, even in many STI clinic settings and laboratories. ${ }^{8} 17$

These TV prevalence rates by PCR are higher than previously reported rates of $<1 \%$ in other urban Australian STI clinic studies, sampling CSWs using traditional diagnostic methods. ${ }^{10} 11$ This difference could signal a true rise in TV prevalence or be attributed to the more sensitive PCR diagnostic techniques employed here as well as sampling a less clinically homogeneous, highly screened population than those represented in previous literature. ${ }^{11}$ However, due to the relatively low population prevalence of TV, the positive predictive value of these PCR findings may be reduced. On the other hand, these PCR data may potentially underestimate the true prevalence due to the strict inclusion criteria of the parent study protocol and retrospective PCR testing of stored, frozen and thawed specimens. Cervical specimens were used for this study, but the performance of cervical and vaginal specimens for TV PCR testing has been shown to be comparable. ${ }^{18}$

An important finding in our study was the high proportion (11/ $17, p=0.003$ ) of women with TV who were of culturally and linguistically diverse backgrounds. Urban society in Australia, as in many other industrialised countries, is very culturally diverse with a highly mobile population. Accordingly, sexual networks, particularly of migrant and first-generation Australians often include consorts who come from or travel to populations with higher TV prevalence. We found the symptom of dysuria to be significantly associated with women with TV, highlighting the need for clinicians to consider the possibility of TV especially in the absence of other causes of dysuria. We did not find any association between TV and BV, although others have. ${ }^{8} \mathrm{BV}$ may mask the presence of TV. Standard BV treatment with nitroimidazoles will effectively eradicate coexistent TV infection, but partners of women with concomitant BV and TV will remain untreated, potentially facilitating the persistence and spread of TV.

Cervicitis was associated with TV (RR 1.66, $p=0.034)$ supporting the role of TV in the aetiology of cervicitis. Nongonococcal, non-chlamydial cervicitis or non-specific cervicitis comprises a major proportion of reported cervicitis, ${ }^{56}$ and TV

\section{Key messages}

We report a higher than previously reported prevalence of Trichomonas vaginalis (TV) of $4.8 \%$ by PCR methods in an urban Australian sexually transmitted infection (STI) clinic setting.

- TV is likely underdiagnosed in urban STI clinic settings using only traditional methods of detection. This may presage reemergence with important Public Health consequences.

- We find TV is significantly associated with women of culturally and linguistically diverse backgrounds, and cervicitis is significantly associated with TV (RR 1.66, $\mathrm{p}=0.034$ ).

- TV warrants a higher profile. Priority should be given to the development of standardised molecular TV detection techniques for inclusion in routine STI testing.

has been recently recognised as an aetiological agent in this condition. ${ }^{5}$ Molecular testing for TV in STI populations is likely to shed further light on the aetiology of non-specific cervicitis in women, a current area of research need.

Coinfection with another STI, including TV, remains a significant risk factor for HIV acquisition. ${ }^{7}$ Women increasingly carry the burden of the HIV epidemic in many developing and westernised countries. ${ }^{19}$ As many screening programmes are targeting younger women, older groups of sexually active women where TV predominates ${ }^{9}$ may be overlooked, potentially contributing to the TV reservoir in the community. The recent rise in gonorrhoea in women in some industrialised nations and noted in our own region ${ }^{20}$ may indicate relaxation of safer sex practices, possibly presaging a rise in STIs with re-establishment in these sexually active populations and increased opportunity for HIV transmission.

If not specifically sought, TV will not be detected. Presently sensitive, standardised detection methods for TV are seldom commercially available, relying on in-house laboratory molecular methods. This, coupled with the low profile of TV, has led to infrequent uptake of any testing for TV in many clinical settings. Our findings suggest that when sensitive diagnostic tests are routinely or selectively applied, TV may be found to be more prevalent than previously thought. These concerns echo those of others ${ }^{27-91621}$ that priority be given to developing standardised, sensitive, cost-effective techniques for TV detection.

In summary, we report a higher than previously reported prevalence of TV, by PCR testing, in an urban Australian STI clinic population. We suggest that the current low profile of TV and the variable application of insensitive tests for case detection have led to underdiagnosis of TV. In turn, this could facilitate a re-emergence of TV, particularly in culturally diverse urban populations. Trichomoniasis has the potential to impact significantly on reproductive health of women and increase HIV transmission. Accordingly, TV warrants a higher profile in urban settings in developed countries. With this demonstration of higher than expected TV prevalence, a call is justified for the development and application of standardised PCR TV-detection techniques to facilitate case-finding, surveillance and continued control of this STI.

Acknowledgements Thanks to the staff and patients of the Short Street Clinic, St George Hospital, and RPA Sexual Health, RPA Hospital, Sydney, Australia, and C McPherson, Laboratory Scientist, SEALS (South Eastern Area laboratory Services) for quality assurance of the Gram-stain microscopy case definition of cervicitis. 
Funding Financial support was provided in part by the HIV and AIDS Related Funding Program (HARP) South Eastern Sydney and Illawarra Area Health Service, and MJL was supported in part by a Novartis Research Scholarship.

\section{Competing interests None.}

\section{Patient consent Obtained.}

Ethics approval Ethics approval was provided by the South Eastern Sydney and Illawarra Area Health Service Human Research Ethics Committee and the Sydney South West Area Heath Service Ethics Review Committee (Royal Prince Alfred Hospital Zone)

Contributors MJL and PK: designed and implemented the clinical research project, collected clinical samples and clinical data and supervised designated clinicians in sample and data collection, undertook data entry and statistical analysis and wrote most of the manuscript (excluding lab methods section). ZN, NR, B Rayner, CJM and WDR: assisted with design of the study, processing and storage of samples and undertook the PCR testing of the clinical samples and contributed to writing the manuscript (lab methods). RGC: assisted with the design of the study, choice of statistical methods, and contributed to reviewing the manuscript. KM: Assisted with the database management and statistical analysis and reviewed manuscript.

Provenance and peer review Not commissioned; externally peer reviewed.

\section{REFERENCES}

1. Krieger JN, Alderete JF. Trichomonas vaginalis and trichomoniasis. In: Holmes KK, Sparling PF, Mardh PA, et al, eds. Sexually transmitted diseases 3rd edn. New York: McGraw-Hill, 1999:771-93.

2. Van der Pol B, Williams JA, Orr DP, et al. Prevalence, incidence, natural history and response to treatment of Trichomonas vaginalis infection among adolescent women. J Invest Dermatol 2005;192:2039-44.

3. Cotch MF, Pastorek JG, Nugent RP, et al. Trichomonas vaginalis associated with low birth weight and preterm delivery. The vaginal Infections and Prematurity Study Group. Sex Transm Dis 1997:24:353-60.

4. Cherpes TL, Wiesenfeld HC, Melan MA, et al. The associations between pelvic inflammatory disease, Trichomonas vaginalis infection and positive herpes simplex virus type 2 serology. Sex Transm Dis 2006;12:747-52.

5. Marrazzo JM, Martin D. Management of women with cervicitis. Clin Infect Dis 2007:44(Suppl 3):102-10.

6. Lusk MJ, Konecny P. Cervicitis-A review. Curr Opin Infect Dis 2008;21:49-55.
7. McClelland RC, Sangere L, Hassan WM, et al. Infection with Trichomonas vaginalis increases the risk of HIV-1 acquisition. J Invest Dermatol 2007;195:698-702.

8. Sena AC, William CM, Hobbs MM, et al. Trichomonas vaginalis infection in male sexual partners: implications for diagnosis, treatment and prevention. Clin Infect Dis 2007:44:13-22.

9. Bowden F, Garnett GP. Trichomonas vaginalis epidemiology parameterising and analysing a model of treatment interventions. Sex Transm Infect 2000;76:248-56.

10. Marrone J, Fairley CK, Saville M, et al. Temporall associations with declining Trichomonas vaginalis diagnosis rates among women in the state of Victoria, Australia, 1947-2005. Sex Transm Dis 2008;35:572-6.

11. Lee DM, Binger $A$, Hocking J, et al. The incidence of sexually transmitted infections among frequently screened sex workers in a decriminalised and regulated system in Melbourne. Sex Transm Infect 2005;81:434-6.

12. Bowen $\mathbf{F}$, Paterson BA, Mein J, et al. Estimating the prevalence of Trichomonas vaginalis, Chlamydia trachomatis, Neisseria Gonorrhoeae and human papillomavirus in indigenous women in Northern Australia. Sex Transm Infect 1999;75:431-44.

13. Sutton M. Sternberg M, Koumans EH, et al. The prevalence of Trichomonas vaginalis infection among reproductive age women in the United States 2001-2004. Clin Infect Dis 2007; 45:1319-26.

14. Mclver CJ, Rismanto N, Smith C, et al. Multiplex PCR testing detection of higherthan-expected rates of cervical mycoplasma, ureaplasma, and trichomonas and vira agent infections in sexually active Australian women. J Clin Microbiol 2009:47:1358-63.

15. Lawing LF, Hedges SR, Schwebke JR. Detection of trichomoniasis in vaginal and urine specimens from women by culture and PCR. J Clin Microbiol 2000;38:3585-8.

16. Wendel KA, Erbelding EJ, Gaydos CA, et al. Trichomonas vaginalis polymerase chain reaction compared with standard diagnostic and therapeutic protocols for detection and treatment of trichomoniasis. Clin Infect Dis 2002:35:576-80.

17. Dicker LW, Mosure DJ, Steece R, et al. Laboratory tests used in US public health laboratories for sexually transmitted diseases, 2000. Sex Transm Dis 2004:31:259-64

18. Van Der Pol B, Kraft CS, Williams JA. Use of an adaptation of a commercially available PCR assay aimed at diagnosis of Chlamydia and gonorrhoea to detect Trichomonas vaginalis in urogenital specimens. J Clin Microbiol 2006:44:366-73.

19. UNAIDS 2007. AIDS epidemic update Geneva, Switzerland: UNAIDS, 2007

20. McDonald A, ed. Annual surveillance report. HIVIAIDS, viral hepatitis and sexually transmissible infections in Australia Sydney, (NSW): National Centre in HIV Epidemiology and Clinical Research, Sydney, 2009:84.

21. Van Der Pol B. Trichomonas vaginalis infection: the most prevalent non viral sexually transmitted infection receives the least public attention. Clin Infect Dis 2007;44:23-5. 